TITLE:

\title{
Smoothing approach to Nash equilibrium formulations for a class of equilibrium problems with shared complementarity constraints
}

\section{AUTHOR(S):}

Hu, Ming; Fukushima, Masao

\section{CITATION:}

Hu, Ming ...[et al]. Smoothing approach to Nash equilibrium formulations for a class of equilibrium problems with shared complementarity constraints. Computational

Optimization and Applications 2012, 52(2): 415-437

ISSUE DATE:

2012-06

URL:

http://hdl.handle.net/2433/157347

\section{RIGHT:}

The final publication is available at www.springerlink.com; この論文は 出版社版でありません。引用の際には出版社版をご確認ご利用くださ $\omega_{\circ}$; This is not the published version. Please cite only the published version. 


\title{
Smoothing Approach to Nash Equilibrium Formulations for \\ a Class of Equilibrium Problems with Shared \\ Complementarity Constraints *
}

\author{
Ming $\mathrm{Hu}^{\dagger} \quad$ Masao Fukushima ${ }^{\ddagger}$ \\ January 31, 2011 \\ Revised, May 07, 2011
}

\begin{abstract}
The equilibrium problem with equilibrium constraints (EPEC) can be looked on as a generalization of Nash equilibrium problem (NEP) and the mathematical program with equilibrium constraints (MPEC) whose constraints contain a parametric variational inequality or complementarity system. In this paper, we particularly consider a special class of EPECs where a common parametric P-matrix linear complementarity system is contained in all players' strategy sets. After reformulating the EPEC as an equivalent nonsmooth NEP, we use a smoothing method to construct a sequence of smoothed NEPs that approximate the original problem. We consider two solution concepts, global Nash equilibrium and stationary Nash equilibrium, and establish some results about the convergence of approximate Nash equilibria. Moreover we show some illustrative numerical examples.

keywords: Equilibrium problem with equilibrium constraints, Nash equilibrium problem, Pmatrix linear complementarity problem, smoothing approximation, global Nash equilibrium, stationary Nash equilibrium.
\end{abstract}

\section{Introduction}

In the classical Nash equilibrium problem (NEP) [17, 18], all players choose their own strategies simultaneously under their respective constraints and try to minimize their own ob-

\footnotetext{
* This work was supported in part by a Grant-in-Aid for Scientific Research from Japan Society for the Promotion of Science.

${ }^{\dagger}$ School of Mathematics and Physics, Jiangsu University of Science and Technology, Zhenjiang 212003, CHINA. Current address: Department of Applied Mathematics and Physics, Graduate School of Informatics, Kyoto University, Kyoto 606-8501, JAPAN. E-mail: mhu@amp.i.kyoto-u.ac.jp.

${ }_{\ddagger}^{\ddagger}$ Department of Applied Mathematics and Physics, Graduate School of Informatics, Kyoto University, Kyoto 606-8501, JAPAN. E-mail: fuku@i.kyoto-u.ac.jp.
} 
jective functions noncooperatively. In the Stackelberg (single-leader-follower) game [16, 22], there exists a distinctive player (called the leader) who can anticipate the responses of the other players (called the followers) and select his optimal strategy. At the same time, given the leader's strategy, all the followers complete with each other in the Nash noncooperative way.

To deal with the Stackelberg game, one can formulate it as a bilevel optimization problem, which may further be reformulated as a mathematical program with equilibrium constraints (MPEC) [16], where the lower level problem is parametrized by the upper level strategy. As a generalization of the MPEC, the equilibrium problem with equilibrium constraints (EPEC) can be formulated as a problem that consists of several MPECs, for which one seeks an equilibrium point that is achieved when all MPECs are optimized simultaneously. The EPEC can be looked on as a formulation of a noncooperative multi-leader-follower game [20]. Several researchers have presented some practical applications of the EPEC, such as electricity markets $[10,11$, 20]. However, only a few attempts have been made so far to develop numerical methods to solve EPECs, even for a special class of problems.

In the study on optimization, one of important subjects is the approximation of optimization problems. In particular, the notion of epiconvergence of functions plays a fundamental role $[13,21]$. Recently, Gürkan and Pang [9] introduced a new notion of epiconvergence, which is called multi-epiconvergence, to study the approximation of Nash equilibrium problems. It is an extension of the notion of epiconvergence to a sequence of families of functions. By means of the new notion, the authors of [9] presented a sufficient condition to ensure the convergence of approximate global Nash equilibria.

In view of the difficulty in computing global Nash equilibria of EPECs that lack convexity, it is reasonable to consider the stationarity in the players' optimization problems. For example, $\mathrm{Hu}$ and Ralph [11] propose two solution concepts called local Nash equilibrium and Nash stationary equilibrium, which are based on local optimality and stationarity, respectively, in each leader's MPEC.

Inspired by their idea and the recent work of Chen and Fukushima [1], in this paper, we consider a special class of EPECs with shared equilibrium constraints formulated as a linear complementarity system. Under some particular assumptions on the linear complementarity system, we show that the EPEC can be reformulated as an nonsmooth NEP. By means of a smoothing technique, we further construct a sequence of smoothed NEPs to approximate this NEP. We consider two solution concepts, global Nash equilibrium and stationary Nash equilibrium, and establish some results about the convergence of approximate Nash equilibria. Moreover, we present some numerical examples for this special class of EPECs. 
The organization of the paper is as follows. In the next section, we collect some basic definitions and present some preliminary results that will be used later. In Section 3, we introduce the particular EPEC considered in the paper, and reformulate it as a NEP. We also introduce a sequence of NEPs by means of a smoothing technique to approximate this NEP. In Section 4, we show some conditions that ensure the convergence of approximate global Nash equilibria. We further consider the approximation of stationary Nash equilibria and show some corresponding results about convergence in Section 5. Next, in Section 6, we present some numerical examples. Finally, we conclude our paper in Section 7.

Notations: The gradient $\nabla f(x)$ of a differentiable function $f: R^{n} \rightarrow R$ is regarded as a column vector. Furthermore, we denote the $n \times m$ (transposed) Jacobian matrix of a differentiable function $F: R^{n} \rightarrow R^{m}$ at a given point $x$ by $\nabla F(x)$. For a real-valued function $f(x, y)$ with variables $x \in R^{n}$ and $y \in R^{m}$, the partial gradients with respect to $x$ and $y$ are denoted by $\nabla_{x} f(x, y) \in R^{n}$ and $\nabla_{y} f(x, y) \in R^{m}$, respectively. A vector is regarded as a column vector. However, if a vector $x$ is composed of several subvectors $x^{1}, \cdots, x^{n}$, it is denoted, for simplicity of notation, as $\left(x^{1}, \cdots, x^{n}\right)$ instead of $\left(\left(x^{1}\right)^{T}, \cdots,\left(x^{n}\right)^{T}\right)^{T}$, where $T$ denotes transposition. We also use $\mathbb{N}$ to denote the set of natural numbers.

\section{Preliminaries}

\subsection{EPECs with Shared Equilibrium Constraints}

In this subsection, we describe a class of EPECs with shared equilibrium constraints and define global and stationary Nash equilibria of the EPECs.

First, we start with the definitions of solution concepts for NEPs. In a NEP, denoted by $\operatorname{NEP}\left(\hat{\theta}_{\nu}, X^{\nu}\right)_{\nu=1}^{N}$, there are $N$ players labelled by integers $\nu=1, \cdots, N$. Player $\nu$ 's strategy is denoted by vector $x^{\nu} \in R^{n_{\nu}}$ and his cost function $\hat{\theta}_{\nu}(x)$ depends on all players' strategies, which are collectively denoted by the vector $x \in R^{n}$ consisting of subvectors $x^{\nu} \in R^{n_{\nu}}, \nu=$ $1, \cdots, N$, and $n=n_{1}+\cdots+n_{N}$. Player $\nu$ 's strategy set $X^{\nu} \subseteq R^{n_{\nu}}$ is independent of the other players' strategies which are denoted collectively as $x^{-\nu}=\left(x^{1}, \cdots, x^{\nu-1}, x^{\nu+1}, \cdots, x^{N}\right) \in$ $R^{n-n_{\nu}}$. For every fixed but arbitrary $x^{-\nu} \in X^{-\nu} \equiv \prod_{\nu^{\prime}=1, \nu^{\prime} \neq \nu}^{N} X^{\nu^{\prime}}$, player $\nu$ solves the following optimization problem for his own variable $x^{\nu}$ :

$$
\begin{array}{ll}
\underset{x^{\nu}}{\operatorname{minimize}} & \hat{\theta}_{\nu}\left(x^{\nu}, x^{-\nu}\right) \\
\text { subject to } & x^{\nu} \in X^{\nu},
\end{array}
$$

where we write $\hat{\theta}_{\nu}(x)=\hat{\theta}_{\nu}\left(x^{\nu}, x^{-\nu}\right)$ to emphasize the role of $x^{\nu}$ in this problem. A tuple 
of strategies $x^{*}=\left(x^{*, \nu}\right)_{\nu=1}^{N} \in X \equiv \prod_{\nu=1}^{N} X^{\nu}$ is called a global Nash Equilibrium if for all $\nu=1, \cdots, N$,

$$
\hat{\theta}_{\nu}\left(x^{*, \nu}, x^{*,-\nu}\right) \leq \hat{\theta}_{\nu}\left(x^{\nu}, x^{*,-\nu}\right) \quad \forall x^{\nu} \in X^{\nu}
$$

On the other hand, a tuple of strategies $x^{*}$ is called a stationary Nash Equilibrium if for all $\nu=1, \cdots, N, x^{*, \nu}$ is a stationary point for the optimization problem (1) with $x^{-\nu}=x^{*,-\nu}$, where a stationary point means that it satisfies a first-order optimality condition for the problem. Assuming the differentiability of the cost functions $\hat{\theta}_{\nu}$ and the convexity of the strategy sets $X^{\nu}$, a stationary Nash equilibrium is characterized as a tuple $x^{*}=\left(x^{*, \nu}\right)_{\nu=1}^{N} \in X$ that satisfies the following conditions for all $\nu=1, \cdots, N$ :

$$
\nabla_{x^{\nu}} \hat{\theta}_{\nu}\left(x^{*, \nu}, x^{*,-\nu}\right)^{T}\left(x^{\nu}-x^{*, \nu}\right) \geq 0 \quad \forall x^{\nu} \in X^{\nu}
$$

If, in addition, $\hat{\theta}_{\nu}$ are convex with respect to $x^{\nu}$ for all $\nu$, then a stationary Nash equilibrium reduces to a global Nash equilibrium. When $\hat{\theta}_{\nu}$ is nondifferentiable, we need to introduce a more general notion of stationarity, as will be done in Section 5.

Recall that a typical MPEC can be defined as the following optimization problem with two sets of variables, the upper level variables $x \in R^{n}$ and the lower level variables $y \in R^{m}$ :

$$
\begin{aligned}
& \text { minimize } \theta(x, y) \\
& \text { subject to } x \in X, y \in S(x),
\end{aligned}
$$

where $\theta: R^{n+m} \rightarrow R, X \subseteq R^{n}$, and for each $x \in X, S(x)$ is the solution set of an equilibrium problem with parameter $x$, which may be represented as a variational inequality problem (VIP) or a complementarity problem (CP).

As a generalization of the MPEC, the EPEC can be formulated as a problem where several players try to solve their own MPECs simultaneously. In particular, we consider an EPEC where $N$ players share the same equilibrium constraints as follows. For each $\nu=1, \cdots, N$, let $X^{\nu} \subseteq R^{n_{\nu}}$ denote the feasible set of upper level variables of player $\nu$, which is independent of the other rival players' variables. We also denote player $\nu$ 's objective function by $\theta_{\nu}\left(x^{\nu}, x^{-\nu}, y\right)$, which is dependent of his own upper level variable $x^{\nu}$ and the other rival players' upper level variables $x^{-\nu} \in X^{-\nu}=\prod_{\nu^{\prime}=1, \nu^{\prime} \neq \nu}^{N} X^{\nu^{\prime}}$ as well as the common lower level variables denoted by $y \in R^{m}$. We assume that the shared equilibrium constraints are represented as the solution set of a parametric equilibrium problem. Specifically, each player $\nu$ 
solves the following optimization problem:

$$
\begin{array}{ll}
\underset{x^{\nu}, y}{\operatorname{minimize}} & \theta_{\nu}\left(x^{\nu}, x^{-\nu}, y\right) \\
\text { subject to } & x^{\nu} \in X^{\nu}, \\
& y \in S\left(x^{\nu}, x^{-\nu}\right),
\end{array}
$$

where $\theta_{\nu}: R^{n+m} \rightarrow R, X^{\nu} \subseteq R^{n_{\nu}}$, and $S\left(x^{\nu}, x^{-\nu}\right)$ is the solution set of an equilibrium problem with parameter $x$, which may be represented as a VIP or a CP.

\subsection{Epiconvergence}

In this subsection, we present some basic definitions and properties about epiconvergence and multi-epiconvergence of functions. First, we begin with the following definitions.

Definition 2.1. [21] For a function $f: R^{n} \rightarrow R$, the epigraph of $f$ is the set

$$
\text { epi } f=\left\{(x, \alpha) \in R^{n+1} \mid \alpha \geq f(x)\right\} \text {. }
$$

Definition 2.2. [12] A sequence of sets $\left\{C_{k}\right\}_{k \in \mathbb{N}}$, where $C_{k} \subset R^{n}$ for all $k \in \mathbb{N}$, is said to converge, in the Painlevé-Kuratowski sense [21], to a set $C \subset R^{n}$, denoted by $C_{k} \rightarrow C$, if

(a) any cluster point of a sequence $\left\{x^{k}\right\}_{k \in \mathbb{N}}$, where $x^{k} \in C_{k}$ for all $k \in \mathbb{N}$, belongs to $C$;

(b) for each $x \in C$, one can find a sequence $\left\{x^{k}\right\}_{k \in \mathbb{N}}$ such that $x^{k} \in C_{k}$ for every $k \in \mathbb{N}$ and $x^{k} \rightarrow x$.

The theory of set convergence provides a convenient tool to study the approximation issues in optimization. In particular, we can define the concept of epiconvergence through the convergence of epigraphs.

Definition 2.3. [21] A sequence of functions $\left\{f_{k}\right\}_{k \in \mathbb{N}}$ on $X \subset R^{n}$, where $f_{k}: R^{n} \rightarrow R$ for each $k$, is said to epiconverge to a function $f: R^{n} \rightarrow R$ on $X$, when epi $f_{k} \rightarrow$ epi $f$ as $k \rightarrow \infty$.

For checking the epiconvergence, the following proposition is useful.

Proposition 2.4. [21] Let $\left\{f_{k}\right\}_{k \in \mathbb{N}}$ be any sequence of functions on $R^{n}$. Then, $f_{k}$ epiconverges to $f$ on $X \subset R^{n}$ if and only if at each point $x \in X$ one has

$$
\left\{\begin{array}{l}
\liminf _{k \rightarrow \infty} f_{k}\left(x^{k}\right) \geq f(x) \quad \text { for every sequence }\left\{x^{k}\right\} \text { such that } x^{k} \rightarrow x, \\
\underset{k \rightarrow \infty}{\limsup } f_{k}\left(x^{k}\right) \leq f(x) \quad \text { for some sequence }\left\{x^{k}\right\} \text { such that } x^{k} \rightarrow x .
\end{array}\right.
$$

It is easy to see from (4) that we have actually $f_{k}\left(x^{k}\right) \rightarrow f(x)$ for at least one sequence $\left\{x^{k}\right\}_{k \in \mathbb{N}}$ such that $x^{k} \rightarrow x$. If this property holds for any such sequence $\left\{x^{k}\right\}$, we say the sequence of functions $\left\{f_{k}\right\}_{k \in \mathbb{N}}$ converges continuously to function $f$. 
Definition 2.5. [21] A sequence of functions $\left\{f_{k}\right\}_{k \in \mathbb{N}}$ is said to converge continuously to a function $f: R^{n} \rightarrow R$ at a point $x \in X \subset R^{n}$ if $f_{k}\left(x^{k}\right) \rightarrow f(x)$ for any sequence $\left\{x^{k}\right\}_{k \in \mathbb{N}} \subset X$ converging to $x$, and to converge continuously on $X \subset R^{n}$ if this is true at every $x \in X$.

The next proposition gives a sufficient condition for a sequence of functions to epiconverge. Proposition 2.6. [21] If a sequence of functions $\left\{f_{k}\right\}_{k \in \mathbb{N}}$ converges continuously to $f: R^{n} \rightarrow$ $R$ on $X \subset R^{n}$, then it epiconverges to $f$ on $X$.

The concept of epiconvergence plays an essential role in studying approximation issues for optimization problems. To study approximation issues for NEPs, which contain multiple objective functions associated with all players' optimization problems, Gürkan and Pang [9] introduced the concept of multi-epiconvergence for a sequence of families of functions, as a generalization of epiconvergence.

Definition 2.7. [9] A sequence of families of functions $\left\{\left\{f_{\nu, k}\right\}_{\nu=1}^{N}\right\}_{k \in \mathbb{N}}$, where $f_{\nu, k}: R^{n} \rightarrow R$ for each $\nu$ and $k$, is said to multi-epiconverge to the family of functions $\left\{f_{\nu}\right\}_{\nu=1}^{N}$, where $f_{\nu}: R^{n} \rightarrow R$ for each $\nu$, on the set $X=\prod_{\nu=1}^{N} X^{\nu} \subset R^{n}$, if the following two conditions hold for every $\nu=1, \cdots, N$ and every $x=\left(x^{1}, \cdots, x^{N}\right) \in X$ :

(a) For every sequence $\left\{x^{-\nu, k}\right\}_{k \in \mathbb{N}} \subset X^{-\nu}$ converging to $x^{-\nu}$, there exists a sequence $\left\{x^{\nu, k}\right\}_{k \in \mathbb{N}}$ $\subset X^{\nu}$ converging to $x^{\nu}$ such that

$$
\limsup _{k \rightarrow \infty} f_{\nu, k}\left(x^{\nu, k}, x^{-\nu, k}\right) \leq f_{\nu}\left(x^{\nu}, x^{-\nu}\right) .
$$

(b) For every sequence $\left\{x^{k}\right\}_{k \in \mathbb{N}} \subset X$ converging to $x$,

$$
\liminf _{k \rightarrow \infty} f_{\nu, k}\left(x^{k}\right) \geq f_{\nu}(x) .
$$

The following proposition establishes the strong relation between multi-epiconvergence and epiconvergence.

Proposition 2.8. [9] A sequence of families of functions $\left\{\left\{f_{\nu, k}\right\}_{\nu=1}^{N}\right\}_{k \in \mathbb{N}}$ multi-epiconverges to the family of functions $\left\{f_{\nu}\right\}_{\nu=1}^{N}$ on the set $X=\prod_{\nu=1}^{N} X^{\nu}$ if and only if for every $\nu=1, \cdots, N$ and every sequence $\left\{x^{-\nu, k}\right\}_{k \in \mathbb{N}} \subset X^{-\nu}$ converging to some $x^{-\nu, \infty} \in X^{-\nu}$, the sequence of functions $\left\{\psi_{\nu, k}\right\}_{k \in \mathbb{N}}$, where each $\psi_{\nu, k}: R^{n_{\nu}} \rightarrow R$ is defined by

$$
\psi_{\nu, k}\left(x^{\nu}\right)=f_{\nu, k}\left(x^{\nu}, x^{-\nu, k}\right), x^{\nu} \in X^{\nu}
$$

epiconverges to the function $\psi_{\nu, \infty}: R^{n_{\nu}} \rightarrow R$ defined by

$$
\psi_{\nu, \infty}\left(x^{\nu}\right)=f_{\nu}\left(x^{\nu}, x^{-\nu, \infty}\right), x^{\nu} \in X^{\nu}
$$

on the set $X^{\nu}$. 
Under the assumption of multi-epiconvergence, the following proposition shows a sufficient condition for the convergence of approximate Nash equilibria.

Proposition 2.9. [9, Theorem 1] Suppose that the sequence offamilies of functions $\left\{\left\{f_{\nu, k}\right\}_{\nu=1}^{N}\right\}_{k \in \mathbb{N}}$ multi-epiconverges to the family of functions $\left\{f_{\nu}\right\}_{\nu=1}^{N}$ on the set $X=\prod_{\nu=1}^{N} X^{\nu}$. If the sequence $\left\{x^{k}\right\}_{k \in \mathbb{N}}$, where each $x^{k}=\left(x^{\nu, k}\right)_{\nu=1}^{N}$ is a global Nash equilibrium of $N E P\left(f_{\nu, k}, X^{\nu}\right)_{\nu=1}^{N}$, converges to $x^{\infty}=\left(x^{\nu, \infty}\right)_{\nu=1}^{N}$, then $x^{\infty}$ is a global Nash equilibrium of $\operatorname{NEP}\left(f_{\nu}, X^{\nu}\right)_{\nu=1}^{N}$.

\subsection{P-Matrix Linear Complementarity Problem}

The complementarity problem $(\mathrm{CP})$ is to find a vector $x \in R^{n}$ such that

$$
F(x) \geq 0, x \geq 0, F(x)^{T} x \geq 0
$$

where $F: R^{n} \rightarrow R^{n}$ is a continuous function. In particular, when $F$ is an affine function represented by $F(x)=\tilde{M} x+\tilde{q}$, where $\tilde{M} \in R^{n \times n}$ and $\tilde{q} \in R^{n}$, the CP becomes the linear complementarity problem (LCP), which is to find a vector $x \in R^{n}$ such that

$$
\tilde{M} x+\tilde{q} \geq 0, x \geq 0, x^{T}(\tilde{M} x+\tilde{q}) \geq 0 .
$$

We denote this problem as $\operatorname{LCP}(\tilde{M}, \tilde{q})$.

Matrix $\tilde{M} \in R^{n \times n}$ is said to be a P-matrix, if its all principal minors are positive, or equivalently,

$$
\max _{1 \leq i \leq n} x_{i}(\tilde{M} x)_{i}>0 \quad \text { for all } x(\neq 0) \in R^{n} .
$$

The following proposition is well known.

Proposition 2.10. [3] A matrix $\tilde{M} \in R^{n \times n}$ is a P-matrix if and only if $L C P(\tilde{M}, \tilde{q})$ has a unique solution for any vector $\tilde{q} \in R^{n}$.

\section{Equilibrium Problem with Shared P-matrix Linear Complementarity Constraints}

In this paper, we consider the particular class of EPECs, where each player's optimization problem contains a P-matrix linear complementarity constraint that is common to all players. Specifically, for $\nu=1, \cdots, N$, given the other players' strategies $x^{-\nu}$, player $\nu$ solves the 
following problem:

$$
\begin{array}{ll}
\underset{x^{\nu}, y}{\operatorname{minimize}} & \theta_{\nu}\left(x^{\nu}, x^{-\nu}, y\right) \\
\text { subject to } & g^{\nu}\left(x^{\nu}\right) \leq 0, h^{\nu}\left(x^{\nu}\right)=0, \\
& \sum_{\mu=1}^{N} K_{\mu} x^{\mu}+M y+q \geq 0, y \geq 0, \\
& \left(\sum_{\mu=1}^{N} K_{\mu} x^{\mu}+M y+q\right)^{T} y=0,
\end{array}
$$

where functions $\theta_{\nu}: R^{n+m} \rightarrow R$ are continuously differentiable on $R^{n+m}, g^{\nu}: R^{n_{\nu}} \rightarrow R^{s_{\nu}}$ are continuously differentiable and convex functions, and $h^{\nu}: R^{n_{\nu}} \rightarrow R^{t_{\nu}}$ are affine functions. In the following, we let $X^{\nu}$ denote $X^{\nu}=\left\{x^{\nu} \in R^{n_{\nu}} \mid g^{\nu}\left(x^{\nu}\right) \leq 0, h^{\nu}\left(x^{\nu}\right)=0\right\}$, which is convex under the given assumptions on $g^{\nu}$ and $h^{\nu}$. The shared linear complementarity constraints

$$
\sum_{\nu=1}^{N} K_{\nu} x^{\nu}+M y+q \geq 0, y \geq 0,\left(\sum_{\nu=1}^{N} K_{\nu} x^{\nu}+M y+q\right)^{T} y=0
$$

are denoted by $\operatorname{LCP}\left(K_{1}, \cdots, K_{N}, M, q\right)$, where $K_{\nu} \in R^{m \times n_{\nu}}, \nu=1, \cdots, N, M \in R^{m \times m}$, and $q \in R^{m}$. In particular, we assume that $M$ is a $\mathrm{P}$-matrix.

Now we define the concept of global Nash equilibria for EPEC $\{(5)\}_{\nu=1}^{N}$.

Definition 3.1. A tuple of strategies $\left(x^{*}, y^{*}\right)=\left(x^{*, 1}, \cdots, x^{*, N}, y^{*}\right)$ is called a global Nash equilibrium of EPEC $\{(5)\}_{\nu=1}^{N}$, if for each $\nu$, the pair of strategies $\left(x^{*, \nu}, y^{*}\right)$ is a global optimal solution for MPEC (5) with $x^{-\nu}=x^{*,-\nu}$.

By Proposition 2.10, for any fixed $x=\left(x^{\nu}\right)_{\nu=1}^{N}, \operatorname{LCP}\left(K_{1}, \cdots, K_{N}, M, q\right)$ has a unique solution $y$, which is denoted $y(x)$ or $y\left(x^{\nu}, x^{-\nu}\right)$, i.e., $S(x)=\{y(x)\}$. It is well known that the solution function $y(\cdot): R^{n} \rightarrow R^{m}$ of the shared P-matrix linear complementarity problem (6) is piecewise linear with respect to the parameter $x$ [16]. Since every piecewise linear function is globally Lipschitz, we have the following proposition.

Proposition 3.2. [1] There is a positive number $\gamma$ such that

$$
\left\|y(x)-y\left(x^{\prime}\right)\right\| \leq \gamma\left\|x-x^{\prime}\right\| \quad \text { for all } x, x^{\prime} \in R^{n} .
$$

We define the functions $\Theta_{\nu}: R^{n} \rightarrow R$ by

$$
\Theta_{\nu}\left(x^{\nu}, x^{-\nu}\right)=\theta_{\nu}\left(x^{\nu}, x^{-\nu}, y\left(x^{\nu}, x^{-\nu}\right)\right), \nu=1, \cdots, N .
$$

Further, we can reformulate EPEC $\{(5)\}_{\nu=1}^{N}$ as the NEP, denoted by $\operatorname{NEP}\left(\Theta_{\nu}, X^{\nu}\right)_{\nu=1}^{N}$, where each player $\nu$ solves the following problem:

$$
\begin{array}{ll}
\underset{x^{\nu}}{\operatorname{minimize}} & \Theta_{\nu}\left(x^{\nu}, x^{-\nu}\right) \\
\text { subject to } & g^{\nu}\left(x^{\nu}\right) \leq 0, h^{\nu}\left(x^{\nu}\right)=0 .
\end{array}
$$


Since $y(\cdot)$ is not differentiable, problem (7) is a nonsmooth optimization problem for each $\nu$.

Recall that $\operatorname{NEP}\left(\Theta_{\nu}, X^{\nu}\right)_{\nu=1}^{N}$ is equivalent to $\operatorname{EPEC}\{(5)\}_{\nu=1}^{N}$ thanks to the property of Pmatrix. This is made precisely in the following proposition.

Proposition 3.3. If $x^{*}$ is a global Nash equilibrium of $N E P\left(\Theta_{\nu}, X^{\nu}\right)_{\nu=1}^{N}$, then $\left(x^{*}, y\left(x^{*}\right)\right)$ is a global Nash equilibrium of EPEC $\{(5)\}_{\nu=1}^{N}$. Conversely, if $\left(x^{*}, y\left(x^{*}\right)\right)$ is a global Nash equilibrium of EPEC $\{(5)\}_{\nu=1}^{N}$, then $x^{*}$ is a global Nash equilibrium of $N E P\left(\Theta_{\nu}, X^{\nu}\right)_{\nu=1}^{N}$.

Proof. To prove the first half, suppose to the contrary that $\left(x^{*}, y\left(x^{*}\right)\right)$ is not a global Nash equilibrium of EPEC $\{(5)\}_{\nu=1}^{N}$. Then there exist an index $\nu$ as well as vectors $\tilde{x}^{\nu}$ and $\tilde{y}$ such that

$$
\theta_{\nu}\left(\tilde{x}^{\nu}, x^{*,-\nu}, \tilde{y}\right)<\theta_{\nu}\left(x^{*, \nu}, x^{*,-\nu}, y\left(x^{*, \nu}, x^{*,-\nu}\right)\right),
$$

and $\tilde{x}^{\nu} \in X^{\nu}, \tilde{y} \in S\left(\tilde{x}^{\nu}, x^{*,-\nu}\right)$. Since the solution set $S\left(\tilde{x}^{\nu}, x^{*,-\nu}\right)$ is a singleton, we have $\tilde{y}=y\left(\tilde{x}^{\nu}, x^{*,-\nu}\right)$, and hence

$$
\theta_{\nu}\left(\tilde{x}^{\nu}, x^{*,-\nu}, y\left(\tilde{x}^{\nu}, x^{*,-\nu}\right)\right)<\theta_{\nu}\left(x^{*, \nu}, x^{*,-\nu}, y\left(x^{*, \nu}, x^{*,-\nu}\right)\right)
$$

that is,

$$
\Theta_{\nu}\left(\tilde{x}^{\nu}, x^{*,-\nu}\right)<\Theta_{\nu}\left(x^{*, \nu}, x^{*,-\nu}\right) .
$$

This contradicts the assumption that $x^{*}$ is global Nash equilibrium of $\operatorname{NEP}\left(\Theta_{\nu}, X^{\nu}\right)_{\nu=1}^{N}$. Thus the first half is proved.

The second half can be proved in a similar manner, and hence the proof is omitted.

It is well known that the complementarity problem (6) can be reformulated as the following system of nonsmooth equations:

$$
\Phi(x, y) \equiv\left(\begin{array}{c}
\phi\left(y_{1},\left(\sum_{\nu=1}^{N} K_{\nu} x^{\nu}+M y+q\right)_{1}\right) \\
\vdots \\
\phi\left(y_{m},\left(\sum_{\nu=1}^{N} K_{\nu} x^{\nu}+M y+q\right)_{m}\right)
\end{array}\right)=0
$$

where $\phi: R^{2} \rightarrow R$ is a function called an NCP function or a complementarity function [5] that satisfies the condition

$$
\phi(a, b)=0 \Longleftrightarrow a \geq 0, b \geq 0, a b=0 .
$$

There are many NCP functions. In this paper, we use the Fischer-Burmeister (FB) function [6] defined by

$$
\phi(a, b)=a+b-\sqrt{a^{2}+b^{2}} .
$$


Smoothing is an effective technique for solving optimization problems and complementarity problems $[4,7,8,19]$. Here, we use a sequence of continuously differentiable functions $\left\{\Phi_{\mu}\right\}$ involving a scalar parameter $\mu>0$ to approximate the nonsmooth function $\Phi: R^{n+m} \rightarrow R^{m}$, where $\Phi_{\mu}: R^{n+m} \rightarrow R^{m}$ is defined by

$$
\Phi_{\mu}(x, y) \equiv\left(\begin{array}{c}
\phi_{\mu}\left(y_{1},\left(\sum_{\nu=1}^{N} K_{\nu} x^{\nu}+M y+q\right)_{1}\right) \\
\vdots \\
\phi_{\mu}\left(y_{m},\left(\sum_{\nu=1}^{N} K_{\nu} x^{\nu}+M y+q\right)_{m}\right)
\end{array}\right) \text {, }
$$

and $\phi_{\mu}: R^{2} \rightarrow R$ is the smoothing Fischer-Burmeister function [14] defined by

$$
\phi_{\mu}(a, b)=a+b-\sqrt{a^{2}+b^{2}+2 \mu^{2}} .
$$

It is easy to see that

$$
\phi_{\mu}(a, b)=0 \Longleftrightarrow a \geq 0, b \geq 0, a b=\mu^{2} .
$$

By virtue of the P-matrix property of $M$, for any fixed $x$, the nonlinear equation

$$
\Phi_{\mu}(x, y)=0
$$

has a unique solution $y$, see [1], which we denote $y_{\mu}(x)$. Compared with the solution function $y(\cdot)$ of the original LCP, the function $y_{\mu}(\cdot)$ has some desirable properties.

Proposition 3.4. [1] There is a positive constant $\kappa$ such that for any $x \in R^{n}$ and $\mu>\mu^{\prime} \geq 0$, we have

$$
\left\|y_{\mu}(x)-y_{\mu^{\prime}}(x)\right\| \leq \kappa\left(\mu-\mu^{\prime}\right) .
$$

Particularly, when $\mu^{\prime}=0$, we have

$$
\left\|y_{\mu}(x)-y(x)\right\| \leq \kappa \mu \text {. }
$$

Proposition 3.5. [1] For any $\mu>0$, the function $y_{\mu}(\cdot)$ is continuously differentiable on $R^{n}$, and there exists a bounded set $\Omega \subset R^{n \times m}$, which is independent of $\mu$, such that $\nabla y_{\mu}(x) \in \Omega$ for all $x \in R^{n}$. Moreover, for any sequence $\left\{x^{k}\right\} \subseteq D_{y}$ converging to $\bar{x}$, where $D_{y}$ denotes the set of points at which $y(\cdot)$ is differentiable, there is a positive sequence $\left\{\mu^{k}\right\}$ tending to 0 such that

$$
\left\{\lim _{k \rightarrow \infty} \nabla y_{\mu^{k}}\left(x^{k}\right)\right\} \subseteq\left\{\lim _{\substack{x \rightarrow \bar{x} \\ x \in D y}} \nabla y(x)\right\} \subseteq \partial y(\bar{x}),
$$

where $\partial y(\bar{x})$ is the Clarke generalized Jacobian of $y(\cdot)$ at $\bar{x}$.

In view of this result, we assume that, for any $\nu$ and any sequence $\left\{x^{k}\right\} \subseteq D_{y}$ converging to $\bar{x}$,

$$
\left\{\lim _{k \rightarrow \infty} \nabla_{x^{\nu}} y_{\mu^{k}}\left(x^{k}\right)\right\} \subseteq \partial_{x^{\nu}} y(\bar{x})
$$


where $\partial_{x^{\nu}} y(\bar{x})$ denotes the Clarke generalized partial Jacobian with respect to $x^{\nu}$ of $y(\cdot)$ at $\bar{x}$.

For any $\mu>0$, we consider a family of functions $\left\{\widehat{\Theta}_{\nu}\right\}_{\nu=1}^{N}$, where the functions $\widehat{\Theta}_{\nu}(\cdot ; \mu)$ : $R^{n} \rightarrow R$ are defined by

$$
\widehat{\Theta}_{\nu}\left(x^{\nu}, x^{-\nu} ; \mu\right)=\theta_{\nu}\left(x^{\nu}, x^{-\nu}, y_{\mu}\left(x^{\nu}, x^{-\nu}\right)\right), \nu=1, \cdots, N
$$

By Proposition 3.5, the functions $\widehat{\Theta}_{\nu}(\cdot ; \mu)$ are continuously differentiable for any $\mu>0$. Let $\left\{\mu^{k}\right\}_{k \in \mathbb{N}} \subset R$ be an arbitrary positive sequence such that $\mu^{k} \rightarrow 0$. Then we define a sequence of families of functions $\left\{\left\{\Theta_{\nu, k}\right\}_{\nu=1}^{N}\right\}_{k \in \mathbb{N}}$ by

$$
\Theta_{\nu, k}\left(x^{\nu}, x^{-\nu}\right)=\widehat{\Theta}_{\nu}\left(x^{\nu}, x^{-\nu} ; \mu^{k}\right), \nu=1, \cdots, N \text {. }
$$

As an approximation to the $\operatorname{NEP}\left(\Theta_{\nu}, X^{\nu}\right)_{\nu=1}^{N}$, we may consider the sequence of NEPs, denoted by $\left\{\operatorname{NEP}\left(\Theta_{\nu, k}, X^{\nu}\right)_{\nu=1}^{N}\right\}_{k \in \mathbb{N}}$, where each player $\nu$ solves the following smooth optimization problem:

$$
\begin{array}{ll}
\underset{x^{\nu}}{\operatorname{minimize}} & \Theta_{\nu, k}\left(x^{\nu}, x^{-\nu}\right) \\
\text { subject to } & g^{\nu}\left(x^{\nu}\right) \leq 0, h^{\nu}\left(x^{\nu}\right)=0 .
\end{array}
$$

\section{Convergence of Approximate Global Nash Equilibria}

In this section, we focus on the convergence of global Nash equilibria for the sequence $\left\{\operatorname{NEP}\left(\Theta_{\nu, k}, X^{\nu}\right)_{\nu=1}^{N}\right\}_{k \in \mathbb{N}}$, where functions $\Theta_{\nu, k}$ are defined by (10) for a positive sequence $\left\{\mu^{k}\right\}$ such that $\mu^{k} \rightarrow 0$. We start with the property of the sequence of functions $\left\{y_{\mu^{k}}(\cdot)\right\}$.

Lemma 4.1. The sequence of functions $\left\{y_{\mu^{k}}(\cdot)\right\}_{k \in \mathbb{N}}$ converges continuously to function $y(\cdot)$ on $X$.

Proof. By the definition of continuous convergence, we need to show that $y_{\mu^{k}}\left(x^{k}\right) \rightarrow y(x)$ for any sequence $\left\{x^{k}\right\}_{k \in \mathbb{N}} \subset X$ converging to $x \in X$. By Propositions 3.2 and 3.4,

$$
\left\|y_{\mu^{k}}\left(x^{k}\right)-y(x)\right\| \leq\left\|y_{\mu^{k}}\left(x^{k}\right)-y\left(x^{k}\right)\right\|+\left\|y\left(x^{k}\right)-y(x)\right\| \leq \kappa \mu^{k}+\gamma\left\|x^{k}-x\right\|,
$$

and hence we have $y_{\mu^{k}}\left(x^{k}\right) \rightarrow y(x)$ as $k \rightarrow \infty$.

Next we show that the sequence of families of functions $\left\{\left\{\Theta_{\nu, k}\right\}_{\nu=1}^{N}\right\}_{k \in \mathbb{N}}$ multi-epiconverges to $\left\{\Theta_{\nu}\right\}_{\nu=1}^{N}$ on $X$. To this end, let $x \in X$ be an arbitrary point and let $\left\{x^{k}\right\} \subset X$ be an arbitrary sequence converging to $x$. Then, for each $\nu$, we define the functions $\Psi_{\nu, k}: R^{n_{\nu}} \rightarrow R$ and $\Psi_{\nu}: R^{n_{\nu}} \rightarrow R$ as follows:

$$
\Psi_{\nu, k}(\cdot)=\Theta_{\nu, k}\left(\cdot, x^{-\nu, k}\right)
$$


and

$$
\Psi_{\nu}(\cdot)=\Theta_{\nu}\left(\cdot, x^{-\nu}\right)
$$

Then we have the following lemmas about functions $\Psi_{\nu, k}$ and $\Psi_{\nu}$.

Lemma 4.2. For each $\nu$ and $k$, the functions $\Psi_{\nu}$ and $\Psi_{\nu, k}$ are continuous on $X$.

Proof. Since $\Psi_{\nu, k}(\cdot)=\Theta_{\nu, k}\left(\cdot, x^{-\nu, k}\right)=\theta_{\nu}\left(\cdot, x^{-\nu, k}, y_{\mu^{k}}\left(\cdot, x^{-\nu, k}\right)\right)$ and $\Psi_{\nu}(\cdot)=\Theta_{\nu}\left(\cdot, x^{-\nu}\right)=$ $\theta_{\nu}\left(\cdot, x^{-\nu}, y\left(\cdot, x^{-\nu}\right)\right)$, the continuity of these functions follows from the continuity of functions $\theta_{\nu}(\cdot), y_{\mu^{k}}(\cdot)$ and $y(\cdot)$.

Lemma 4.3. For each $\nu$, the sequence of functions $\left\{\Psi_{\nu, k}\right\}_{k \in \mathbb{N}}$ converges continuously to function $\Psi_{\nu}$ on $X^{\nu}$.

Proof. By the definition of continuous convergence, we need to show that for any $\tilde{x}^{\nu} \in X^{\nu}$ and any sequence $\left\{\tilde{x}^{\nu, k}\right\}_{k \in \mathbb{N}} \in X^{\nu}$ such that $\tilde{x}^{\nu, k} \rightarrow \tilde{x}^{\nu}$, we have $\Psi_{\nu, k}\left(\tilde{x}^{\nu, k}\right) \rightarrow \Psi_{\nu}\left(\tilde{x}^{\nu}\right)$, i.e., $\theta_{\nu}\left(\tilde{x}^{\nu, k}, x^{-\nu, k}, y_{\mu^{k}}\left(\tilde{x}^{\nu, k}, x^{-\nu, k}\right)\right) \rightarrow \theta_{\nu}\left(\tilde{x}^{\nu}, x^{-\nu}, y\left(\tilde{x}^{\nu}, x^{-\nu}\right)\right)$. Since $x^{-\nu, k} \rightarrow x^{-\nu}$ and $\mu^{k} \rightarrow 0$, it follows from Lemma 4.1 that $y_{\mu^{k}}\left(\tilde{x}^{\nu, k}, x^{-\nu, k}\right) \rightarrow y\left(\tilde{x}^{\nu}, x^{-\nu}\right)$, and hence $\theta_{\nu}\left(\tilde{x}^{\nu, k}, x^{-\nu, k}, y_{\mu^{k}}\left(\tilde{x}^{\nu, k}\right.\right.$, $\left.\left.x^{-\nu, k}\right)\right) \rightarrow \theta_{\nu}\left(\tilde{x}^{\nu}, x^{-\nu}, y\left(\tilde{x}^{\nu}, x^{-\nu}\right)\right)$ by the continuity of $\theta_{\nu}$.

By Proposition 2.6, Lemma 4.3 means that the sequence of functions $\left\{\Psi_{\nu, k}\right\}_{k \in \mathbb{N}}$ epiconverges to the function $\Psi_{\nu}$. Thus we have the following lemma.

Lemma 4.4. The sequence of families of functions $\left\{\left\{\Theta_{\nu, k}\right\}_{\nu=1}^{N}\right\}_{k \in \mathbb{N}}$ multi-epiconverges to the family of functions $\left\{\Theta_{\nu}\right\}_{\nu=1}^{N}$.

Proof. By Proposition 2.8, it is sufficient to show that for every $\nu=1, \cdots, N$ and every sequence $\left\{x^{-\nu, k}\right\}_{k \in \mathbb{N}} \subset X^{-\nu}$ converging to some $x^{-\nu} \in X^{-\nu}$, the sequence of functions $\left\{\Psi_{\nu, k}\right\}_{k \in \mathbb{N}}$ defined by (12) epiconverges to the function $\Psi_{\nu}$ defined by (13). It is true as mentioned just after Lemma 4.3.

Based on this result, we establish convergence of the approximate global Nash equilibria.

Theorem 4.5. Let a sequence $\left\{x^{k}\right\}_{k \in \mathbb{N}}$ be such that each $x^{k}$ is a global Nash equilibrium of $\operatorname{NEP}\left(\Theta_{\nu, k}, X^{\nu}\right)_{\nu=1}^{N}$. If $\left\{x^{k}\right\}_{k \in \mathbb{N}}$ converges to $x^{*}$, then $x^{*}$ is a global Nash equilibrium of the $\operatorname{NEP}\left(\Theta_{\nu}, X^{\nu}\right)_{\nu=1}^{N}$.

Proof. Follows immediately from Lemma 4.4 and Proposition 2.9. 


\section{Convergence of Approximate Stationary Nash Equilibria}

In this section, we further investigate the behavior of a sequence of stationary Nash equilibria for $\left\{\operatorname{NEP}\left(\Theta_{\nu, k}, X^{\nu}\right)_{\nu=1}^{N}\right\}_{k \in \mathbb{N}}$. This is important from the numerical point of view, since in practice we may only expect to compute a stationary point of each player's optimization problem.

To this end, we need to introduce some stationary concepts which are associated with the $\operatorname{NEP}\left(\Theta_{\nu}, X^{\nu}\right)_{\nu=1}^{N}$. First, we notice that the function $y(\cdot)$ is directionally differentiable everywhere [3, Theorem 7.4.2]. Then the directional derivative of $y\left(\cdot, x^{-\nu}\right)$ at $x^{\nu} \in R^{n_{\nu}}$ in the direction $d^{\nu} \in R^{n_{\nu}}$, denoted by $y_{x^{\nu}}^{\prime}\left(x^{\nu}, x^{-\nu} ; d^{\nu}\right) \in R^{m}$, is defined as follows:

$$
y_{x^{\nu}}^{\prime}\left(x^{\nu}, x^{-\nu} ; d^{\nu}\right)=\lim _{t \downarrow 0} \frac{y\left(x^{\nu}+t d^{\nu}, x^{-\nu}\right)-y\left(x^{\nu}, x^{-\nu}\right)}{t} .
$$

Based on the concepts of B-stationary point and C-stationary point for a nonsmooth optimization problem, we introduce the corresponding concepts for a nonsmooth NEP as follows.

We call $x \in X$ a Bouligand stationary (B-stationary) Nash equilibrium [1] of $\operatorname{NEP}\left(\Theta_{\nu}, X^{\nu}\right)_{\nu=1}^{N}$ if for each $\nu=1, \cdots, N$,

$$
\begin{aligned}
\Theta_{\nu, x^{\nu}}^{\prime}\left(x^{\nu}, x^{-\nu} ; d^{\nu}\right)= & \nabla_{x^{\nu}} \theta_{\nu}\left(x^{\nu}, x^{-\nu}, y\left(x^{\nu}, x^{-\nu}\right)\right)^{T} d^{\nu} \\
& +\nabla_{y} \theta_{\nu}\left(x^{\nu}, x^{-\nu}, y\left(x^{\nu}, x^{-\nu}\right)\right)^{T} y_{x^{\nu}}^{\prime}\left(x^{\nu}, x^{-\nu} ; d^{\nu}\right) \geq 0
\end{aligned}
$$

for all $d^{\nu} \in \mathcal{T}\left(x^{\nu} ; X^{\nu}\right)$. Here $\mathcal{T}\left(x^{\nu} ; X^{\nu}\right)$ denotes the tangent cone [5] of $X^{\nu}$ at $x^{\nu}$, which consists of all vectors $d^{\nu} \in R^{n_{\nu}}$, called tangent vectors to $X^{\nu}$ at $x^{\nu}$, satisfying the condition that there exist a sequence of vectors $\left\{x^{\nu, k}\right\} \subseteq X^{\nu}$ and a sequence of positive scalars $\left\{\tau_{k}^{\nu}\right\}$ such that

$$
\lim _{k \rightarrow \infty} x^{\nu, k}=x^{\nu}, \lim _{k \rightarrow \infty} \tau_{k}^{\nu}=0, \lim _{k \rightarrow \infty} \frac{x^{\nu, k}-x^{\nu}}{\tau_{k}^{\nu}}=d^{\nu} .
$$

Associated with the players' optimization problems, we introduce the following MangasarianFromovitz constraint qualification (MFCQ): For each $\nu=1, \cdots, N,\left\{\nabla h_{j}^{\nu}\left(x^{\nu}\right) \mid j=1, \cdots, s_{\nu}\right\}$ is a linearly independent set and there exists a vector $d^{\nu} \in R^{n_{\nu}}$ such that

$$
\nabla g_{i}^{\nu}\left(x^{\nu}\right)^{T} d^{\nu}<0, i \in \mathcal{I}\left(x^{\nu}\right), \nabla h_{j}^{\nu}\left(x^{\nu}\right)^{T} d^{\nu}=0, j=1, \cdots, s_{\nu}
$$

where $\mathcal{I}\left(x^{\nu}\right)=\left\{i \mid g_{i}^{\nu}\left(x^{\nu}\right)=0\right\}$.

Under the MFCQ, the tangent cone $\mathcal{T}\left(x^{\nu} ; X^{\nu}\right)$ can be represented precisely as follows:

$$
\mathcal{T}\left(x^{\nu} ; X^{\nu}\right)=\left\{d^{\nu} \mid \nabla g_{i}^{\nu}\left(x^{\nu}\right)^{T} d^{\nu} \leq 0, i \in \mathcal{I}\left(x^{\nu}\right), \nabla h_{j}^{\nu}\left(x^{\nu}\right)^{T} d^{\nu}=0, j=1, \cdots, s_{\nu}\right\}
$$

Then, $x=\left(x^{\nu}\right)_{\nu=1}^{N} \in R^{n}$ is called a Clarke stationary (C-stationary) Nash equilibrium for $\operatorname{NEP}\left(\Theta_{\nu}, X^{\nu}\right)_{\nu=1}^{N}$ if for each $\nu, x^{\nu} \in R^{n_{\nu}}$ together with some Lagrange multipliers $\left(\lambda^{\nu}, \eta^{\nu}\right) \in$ 
$R^{s_{\nu}} \times R^{t_{\nu}}$ satisfies the following Karush-Kuhn-Tucker (KKT) conditions:

$$
\begin{array}{r}
\partial_{x^{\nu}} \Theta_{\nu}\left(x^{\nu}, x^{-\nu}\right)+\nabla g^{\nu}\left(x^{\nu}\right) \lambda^{\nu}+\nabla h^{\nu}\left(x^{\nu}\right) \eta^{\nu} \ni 0, \\
g^{\nu}\left(x^{\nu}\right) \leq 0, \lambda^{\nu} \geq 0,\left(\lambda^{\nu}\right)^{T} g^{\nu}\left(x^{\nu}\right)=0, \\
h^{\nu}\left(x^{\nu}\right)=0,
\end{array}
$$

where $\partial_{x^{\nu}} \Theta_{\nu}\left(x^{\nu}, x^{-\nu}\right)$ is the Clarke generalized gradient of $\Theta_{\nu}\left(\cdot, x^{-\nu}\right)$ at $x^{\nu}$.

Since function $y_{\mu}(\cdot)$ is continuously differentiable on $R^{n}$ for any $\mu>0$, the B-stationarity condition for the $\operatorname{NEP}\left(\Theta_{\nu, k}, X^{\nu}\right)_{\nu=1}^{N}$ can be written as follows:

$$
\begin{aligned}
\nabla_{x^{\nu}} \Theta_{\nu, k}\left(x^{\nu}, x^{-\nu}\right)^{T} d^{\nu}= & \nabla_{x^{\nu}} \theta_{\nu, k}\left(x^{\nu}, x^{-\nu}, y_{\mu^{k}}\left(x^{\nu}, x^{-\nu}\right)\right)^{T} d^{\nu} \\
& +\nabla_{y} \theta_{\nu, k}\left(x^{\nu}, x^{-\nu}, y_{\mu^{k}}\left(x^{\nu}, x^{-\nu}\right)\right)^{T} \nabla_{x^{\nu}} y_{\mu^{k}}\left(x^{\nu}, x^{-\nu}\right)^{T} d^{\nu} \geq 0
\end{aligned}
$$

for all $d^{\nu} \in \mathcal{T}\left(x^{\nu} ; X^{\nu}\right)$.

Moreover, for each $k$, we say $x=\left(x^{\nu}\right)_{\nu=1}^{N} \in R^{n}$ is a KKT point of $\operatorname{NEP}\left(\Theta_{\nu, k}, X^{\nu}\right)_{\nu=1}^{N}$, if for each $\nu, x^{\nu} \in R^{n_{\nu}}$ together with some $\left(\lambda^{\nu}, \eta^{\nu}\right) \in R^{s_{\nu}} \times R^{t_{\nu}}$ satisfies the following system:

$$
\begin{aligned}
\nabla_{x^{\nu}} \Theta_{\nu, k}\left(x^{\nu}, x^{-\nu}\right)+\nabla g^{\nu}\left(x^{\nu}\right) \lambda^{\nu}+\nabla h^{\nu}\left(x^{\nu}\right) \eta^{\nu} & =0 \\
g^{\nu}\left(x^{\nu}\right) \leq 0, \lambda^{\nu} \geq 0,\left(\lambda^{\nu}\right)^{T} g^{\nu}\left(x^{\nu}\right) & =0 \\
h^{\nu}\left(x^{\nu}\right) & =0 .
\end{aligned}
$$

Note that under the MFCQ, conditions (16) and (17) are equivalent.

Now we establish the following result about the convergence of a sequence of KKT points of smoothed problems $\left\{\operatorname{NEP}\left(\Theta_{\nu, k}, X^{\nu}\right)_{\nu=1}^{N}\right\}_{k \in \mathbb{N}}$ to a C-stationary Nash equilibrium of $\operatorname{NEP}\left(\Theta_{\nu}, X^{\nu}\right)_{\nu=1}^{N}$.

Theorem 5.1. Suppose that the feasible set $X=\left\{x=\left(x^{\nu}\right)_{\nu=1}^{N} \in R^{n} \mid g^{\nu}\left(x^{\nu}\right) \leq 0, h^{\nu}\left(x^{\nu}\right)=\right.$ $0, \nu=1, \cdots, N\}$ of $\operatorname{NEP}\left(\Theta_{\nu}, X^{\nu}\right)_{\nu=1}^{N}$ is bounded and the MFCQ holds at any $x \in X$. Let $\left\{x^{k}\right\}_{k \in \mathbb{N}}$ be a sequence of B-stationary Nash equilibria of the family of smoothed problems $\left\{N E P\left(\Theta_{\nu, k}, X^{\nu}\right)_{\nu=1}^{N}\right\}_{k \in \mathbb{N}}$. Moreover, we assume that (9) holds. Then every accumulation point $\bar{x}$ of the sequence $\left\{x^{k}\right\}_{k \in \mathbb{N}}$ is a C-stationary Nash equilibrium of $\operatorname{NEP}\left(\Theta_{\nu}, X^{\nu}\right)_{\nu=1}^{N}$.

Proof. Since under the MFCQ, a B-stationary Nash equilibrium and a KKT point are equivalent for $\operatorname{NEP}\left(\Theta_{\nu, k}, X^{\nu}\right)_{\nu=1}^{N}$. Therefore, for each $k$ and $\nu$, along with some corresponding Lagrange multipliers pair $\left(\lambda^{\nu, k}, \eta^{\nu, k}\right)$, we have from (17)

$$
\begin{aligned}
& \nabla_{x^{\nu}} y_{\mu^{k}}\left(x^{\nu, k}, x^{-\nu, k}\right) \nabla_{y} \theta_{\nu}\left(x^{\nu, k}, x^{-\nu, k}, y_{\mu^{k}}\left(x^{\nu, k}, x^{-\nu, k}\right)\right) \\
&+\nabla_{x^{\nu}} \theta_{\nu}\left(x^{\nu, k}, x^{-\nu, k}, y_{\mu^{k}}\left(x^{\nu, k}, x^{-\nu, k}\right)\right)+\nabla g^{\nu}\left(x^{\nu, k}\right) \lambda^{\nu, k}+\nabla h^{\nu}\left(x^{\nu, k}\right) \eta^{\nu, k}=0, \\
& g^{\nu}\left(x^{\nu, k}\right) \leq 0, \lambda^{\nu, k} \geq 0,\left(\lambda^{\nu, k}\right)^{T} g^{\nu}\left(x^{\nu, k}\right)=0, \\
& h^{\nu}\left(x^{\nu, k}\right)=0 .
\end{aligned}
$$


Note that the sequence $\left\{x^{k}\right\}$ is bounded since the feasible set is bounded. Let $\bar{x}$ be an accumulation point of $\left\{x^{k}\right\}$. For each $\nu=1, \cdots, N$, by the continuity of functions $\nabla_{x^{\nu}} \theta_{\nu}, \nabla_{y} \theta_{\nu}, \nabla g^{\nu}, \nabla h^{\nu}$ and $y(\cdot)$ along with Proposition 3.3, we have

$$
\begin{aligned}
\lim _{k \rightarrow \infty} \nabla_{x^{\nu}} \theta_{\nu}\left(x^{\nu, k}, x^{-\nu, k}, y_{\mu^{k}}\left(x^{\nu, k}, x^{-\nu, k}\right)\right) & =\nabla_{x^{\nu}} \theta_{\nu}\left(\bar{x}^{\nu}, \bar{x}^{-\nu}, y\left(\bar{x}^{\nu}, \bar{x}^{-\nu}\right)\right), \\
\lim _{k \rightarrow \infty} \nabla_{y} \theta_{\nu}\left(x^{\nu, k}, x^{-\nu, k}, y_{\mu^{k}}\left(x^{\nu, k}, x^{-\nu, k}\right)\right) & =\nabla_{y} \theta_{\nu}\left(\bar{x}^{\nu}, \bar{x}^{-\nu}, y\left(\bar{x}^{\nu}, \bar{x}^{-\nu}\right)\right), \\
\lim _{k \rightarrow \infty} \nabla g^{\nu}\left(x^{\nu, k}\right) & =\nabla g^{\nu}\left(\bar{x}^{\nu}\right), \\
\lim _{k \rightarrow \infty} \nabla h^{\nu}\left(x^{\nu, k}\right) & =\nabla h^{\nu}\left(\bar{x}^{\nu}\right) .
\end{aligned}
$$

By (9), for each $\nu$ there exist a matrix $V^{\nu} \in \partial_{x^{\nu}} y(\bar{x})$ and a subsequence of $\left\{x^{k}\right\}$, still denoted by $\left\{x^{k}\right\}$ for simplicity, such that $x^{k} \rightarrow \bar{x}$ and

$$
\lim _{k \rightarrow \infty} \nabla_{x^{\nu}} y_{\mu^{k}}\left(x^{\nu, k}, x^{-\nu, k}\right)=V^{\nu} \in \partial_{x^{\nu}} y\left(\bar{x}^{\nu}, \bar{x}^{-\nu}\right) .
$$

Since the MFCQ holds at $\bar{x}$, it is not difficult to show that the sequence $\left\{\left(\lambda^{k}, \mu^{k}\right)\right\}_{k \in \mathbb{N}}$ is bounded. Without loss of generality, we assume that $\left(x^{k}, \lambda^{k}, \mu^{k}\right) \rightarrow(\bar{x}, \bar{\lambda}, \bar{\mu})$.

Then, for each $\nu=1, \cdots, N$, it follows from (18) that

$$
\begin{aligned}
V^{\nu} \nabla_{y} \theta_{\nu}\left(\bar{x}^{\nu}, \bar{x}^{-\nu}, y\left(\bar{x}^{\nu}, \bar{x}^{-\nu}\right)\right)+ & \nabla_{x^{\nu}} \theta_{\nu}\left(\bar{x}^{\nu}, \bar{x}^{-\nu}, y\left(\bar{x}^{\nu}, \bar{x}^{-\nu}\right)\right) \\
+\nabla g^{\nu}\left(\bar{x}^{\nu}\right) \bar{\lambda}^{\nu}+\nabla h^{\nu}\left(\bar{x}^{\nu}\right) \bar{\eta}^{\nu} & =0, \\
g^{\nu}\left(\bar{x}^{\nu}\right) \leq 0, \bar{\lambda}^{\nu} \geq 0,\left(\bar{\lambda}^{\nu}\right)^{T} g^{\nu}\left(\bar{x}^{\nu}\right) & =0, \\
h^{\nu}\left(\bar{x}^{\nu}\right) & =0 .
\end{aligned}
$$

By the continuous differentiability of function $\theta_{\nu}$ for each $\nu$, the Jacobian chain rule [2, Theorem 2.6.6] yields

$$
\nabla_{x^{\nu}} \theta_{\nu}\left(\bar{x}^{\nu}, \bar{x}^{-\nu}, y\left(\bar{x}^{\nu}, \bar{x}^{-\nu}\right)\right)+\partial_{x^{\nu}} y\left(\bar{x}^{\nu}, \bar{x}^{-\nu}\right) \nabla_{y} \theta_{\nu}\left(\bar{x}^{\nu}, \bar{x}^{-\nu}, y\left(\bar{x}^{\nu}, \bar{x}^{-\nu}\right)\right)=\partial_{x^{\nu}} \Theta_{\nu}\left(\bar{x}^{\nu}, \bar{x}^{-\nu}\right) .
$$

This along with (19) means that (15) holds for all $\nu=1, \cdots, N$ with $(x, \lambda, \mu)=(\bar{x}, \bar{\lambda}, \bar{\mu})$. Therefore, $\bar{x}$ is a C-stationary Nash equilibrium of $\operatorname{NEP}\left(\Theta_{\nu}, X^{\nu}\right)_{\nu=1}^{N}$.

Now we consider some properties about the B-stationary points.

Lemma 5.2. Let $\bar{x} \in X$ be an accumulation point of the sequence $\left\{x^{k}\right\}$, where each $x^{k}$ is a $B$-stationary Nash equilibrium of $N E P\left(\Theta_{\nu, k}, X^{\nu}\right)_{\nu=1}^{N}$. Assume that (9) holds and so does the MFCQ at $\bar{x}$. Then for each $\nu=1, \cdots, N$, there exists a matrix $V^{\nu} \in \partial_{x^{\nu}} y\left(\bar{x}^{\nu}, \bar{x}^{-\nu}\right)$ such that

$$
\nabla_{x^{\nu}} \theta_{\nu}\left(\bar{x}^{\nu}, \bar{x}^{-\nu}, y\left(\bar{x}^{\nu}, \bar{x}^{-\nu}\right)\right)^{T} d^{\nu}+\nabla_{y} \theta_{\nu}\left(\bar{x}^{\nu}, \bar{x}^{-\nu}, y\left(\bar{x}^{\nu}, \bar{x}^{-\nu}\right)\right)^{T}\left(V^{\nu}\right)^{T} d^{\nu} \geq 0
$$

for all $d^{\nu} \in \mathcal{T}\left(\bar{x}^{\nu} ; X^{\nu}\right)$. 
Proof. Since each $x^{k}$ is a B-stationary Nash equilibrium of $\operatorname{NEP}\left(\Theta_{\nu, k}, X^{\nu}\right)_{\nu=1}^{N}$, we have

$$
\begin{aligned}
\nabla_{x^{\nu}} \Theta_{\nu, k}\left(x^{\nu, k}, x^{-\nu, k}\right)^{T} d^{\nu, k}= & \nabla_{x^{\nu}} \theta_{\nu}\left(x^{\nu, k}, x^{-\nu, k}, y_{\mu^{k}}\left(x^{\nu, k}, x^{-\nu, k}\right)\right)^{T} d^{\nu, k} \\
& +\nabla_{y} \theta_{\nu}\left(x^{\nu, k}, x^{-\nu, k}, y_{\mu^{k}}\left(x^{\nu, k}, x^{-\nu, k}\right)\right)^{T} \nabla_{x^{\nu}} y_{\mu^{k}}\left(x^{\nu, k}, x^{-\nu, k}\right)^{T} d^{\nu, k} \\
\geq & 0
\end{aligned}
$$

for all $d^{\nu, k} \in \mathcal{T}\left(x^{\nu, k} ; X^{\nu}\right)$.

For each $\nu=1, \cdots, N$, since $\bar{x}$ is an accumulation point of $\left\{x^{k}\right\}$, by (9), there exist a matrix $V^{\nu} \in \partial_{x^{\nu}} y\left(\bar{x}^{\nu}, \bar{x}^{-\nu}\right)$ and a subsequence of $\left\{x^{\nu, k}\right\}$, still denoted by $\left\{x^{\nu, k}\right\}$ for simplicity, which converges to $\bar{x}^{\nu}$, such that

$$
\lim _{k \rightarrow \infty} \nabla_{x^{\nu}} y_{\mu^{k}}\left(x^{\nu, k}, x^{-\nu, k}\right)=V^{\nu} \in \partial_{x^{\nu}} y\left(\bar{x}^{\nu}, \bar{x}^{-\nu}\right)
$$

Further, for any $d^{\nu} \in \mathcal{T}\left(\bar{x}^{\nu} ; X\right)$, by the MFCQ, there exists a sequence $\left\{d^{\nu, k}\right\}$ such that $d^{\nu, k} \rightarrow$ $d^{\nu}$ as $k \rightarrow \infty$. Then, by passing to the limit $k \rightarrow \infty$ in (16), we can deduce that

$$
\nabla_{x^{\nu}} \theta_{\nu}\left(\bar{x}^{\nu}, \bar{x}^{-\nu}, y\left(\bar{x}^{\nu}, \bar{x}^{-\nu}\right)\right)^{T} d^{\nu}+\nabla_{y} \theta_{\nu}\left(\bar{x}^{\nu}, \bar{x}^{-\nu}, y\left(\bar{x}^{\nu}, \bar{x}^{-\nu}\right)\right)^{T}\left(V^{\nu}\right)^{T} d^{\nu} \geq 0
$$

Since this holds for all $d^{\nu} \in \mathcal{T}\left(\bar{x}^{\nu} ; X\right)$, the desired result follows.

In the light of Lemma 5.2, we show a convergence result about B-stationary Nash equilibria. To this end, we introduce the following concept.

Definition 5.3. [2] For each $\nu=1, \cdots, N$, function $\Theta_{\nu}: R^{n} \rightarrow R$ is said to be regular if, for all $x^{\nu} \in R^{n_{\nu}}, x^{-\nu} \in R^{n-n_{\nu}}$ and $d^{\nu} \in R^{n_{\nu}}$, the directional derivative $\Theta_{\nu, x^{\nu}}^{\prime}\left(x^{\nu}, x^{-\nu} ; d^{\nu}\right)$ exists and satisfies

$$
\Theta_{\nu, x^{\nu}}^{\prime}\left(x^{\nu}, x^{-\nu} ; d^{\nu}\right)=\limsup _{\substack{z^{\nu} \rightarrow \nu^{\nu} \\ t \downarrow 0}} \frac{\Theta_{\nu}\left(z^{\nu}+t d^{\nu}, x^{-\nu}\right)-\Theta_{\nu}\left(z^{\nu}, x^{-\nu}\right)}{t} .
$$

Theorem 5.4. Suppose that the feasible set $X=\left\{x=\left(x^{\nu}\right)_{\nu=1}^{N} \in R^{n} \mid g^{\nu}\left(x^{\nu}\right) \leq 0, h^{\nu}\left(x^{\nu}\right)=\right.$ $0, \nu=1, \cdots, N\}$ of $\operatorname{NEP}\left(\Theta_{\nu}, X^{\nu}\right)_{\nu=1}^{N}$ is bounded, the MFCQ holds at any $x \in X$, and each objective function $\Theta_{\nu}$ is regular with respect to its own variable $x^{\nu}, \nu=1, \cdots, N$. Let $\left\{x^{k}\right\}$ be a sequence of B-stationary Nash equilibria of $N E P\left(\Theta_{\nu, k}, X^{\nu}\right)_{\nu=1}^{N}$, and assume that (9) holds. Then every accumulation point of $\left\{x^{k}\right\}$ is a B-stationary Nash equilibrium of $N E P\left(\Theta_{\nu}, X^{\nu}\right)_{\nu=1}^{N}$.

Proof. The sequence $\left\{x^{k}\right\}$ is bounded since $X$ is bounded. Let $\bar{x}$ be an arbitrary accumulation point of $\left\{x^{k}\right\}$. Then, by Lemma 5.2, for each $\nu=1, \cdots, N$, there exists a matrix $V^{\nu} \in$ $\partial_{x^{\nu}} y\left(\bar{x}^{\nu}, \bar{x}^{-\nu}\right)$ such that

$$
\nabla_{x^{\nu}} \theta_{\nu}\left(\bar{x}^{\nu}, \bar{x}^{-\nu}, y\left(\bar{x}^{\nu}, \bar{x}^{-\nu}\right)\right)^{T} d^{\nu}+\nabla_{y} \theta_{\nu}\left(\bar{x}^{\nu}, \bar{x}^{-\nu}, y\left(\bar{x}^{\nu}, \bar{x}^{-\nu}\right)\right)^{T}\left(V^{\nu}\right)^{T} d^{\nu} \geq 0
$$


for all $d^{\nu} \in \mathcal{T}\left(\bar{x}^{\nu} ; X^{\nu}\right)$. Moreover, by the Jacobian chain rule [2, Theorem 2.6.6], we have

$$
\nabla_{x^{\nu}} \theta_{\nu}\left(\bar{x}^{\nu}, \bar{x}^{-\nu}, y\left(\bar{x}^{\nu}, \bar{x}^{-\nu}\right)\right)+V^{\nu} \nabla_{y} \theta_{\nu}\left(\bar{x}^{\nu}, \bar{x}^{-\nu}, y\left(\bar{x}^{\nu}, \bar{x}^{-\nu}\right)\right) \in \partial_{x^{\nu}} \Theta_{\nu}\left(\bar{x}^{\nu}, \bar{x}^{-\nu}\right)
$$

Further, by the regularity of $\Theta_{\nu}$ and [2, Proposition 2.1.2 (b)], we also have

$$
\Theta_{\nu, x^{\nu}}^{\prime}\left(\bar{x}^{\nu}, \bar{x}^{-\nu} ; d^{\nu}\right)=\max \left\{\left(\xi^{\nu}\right)^{T} d^{\nu}: \xi^{\nu} \in \partial_{x^{\nu}} \Theta_{\nu}\left(\bar{x}^{\nu}, \bar{x}^{-\nu}\right)\right\}
$$

for all $d^{\nu} \in \mathcal{T}\left(\bar{x}^{\nu} ; X^{\nu}\right)$.

Therefore, in view of (20) and (21), we can deduce that

$$
\begin{aligned}
\Theta_{\nu, x^{\nu}}^{\prime}\left(\bar{x}^{\nu}, \bar{x}^{-\nu} ; d^{\nu}\right) \geq & \nabla_{x^{\nu}} \theta_{\nu}\left(\bar{x}^{\nu}, \bar{x}^{-\nu}, y\left(\bar{x}^{\nu}, \bar{x}^{-\nu}\right)\right)^{T} d^{\nu} \\
& +\nabla_{y} \theta_{\nu}\left(\bar{x}^{\nu}, \bar{x}^{-\nu}, y\left(\bar{x}^{\nu}, \bar{x}^{-\nu}\right)\right)^{T}\left(V^{\nu}\right)^{T} d^{\nu} \geq 0
\end{aligned}
$$

for all $d^{\nu} \in \mathcal{T}\left(\bar{x}^{\nu} ; X^{\nu}\right)$. Since this holds for all $\nu=1, \cdots, N, \bar{x}$ is a B-stationary Nash equilibrium of $\operatorname{NEP}\left(\Theta_{\nu}, X^{\nu}\right)_{\nu=1}^{N}$.

\section{Numerical Experiments}

In this section, we show some numerical results for the class of EPECs described in Section 3. Specifically, we consider the following EPEC with two players, which contains a shared P-matrix linear complementarity constraint parameterized by the upper level variables $x=$ $\left(x^{\mathrm{I}}, x^{\mathrm{II}}\right) \in R^{n}:$

Player I's problem:

$$
\begin{array}{cl}
\underset{x^{\mathrm{I}}, y}{\operatorname{minimize}} & \frac{1}{2}\left(x^{\mathrm{I}}\right)^{T} H_{\mathrm{I}} x^{\mathrm{I}}+\left(x^{\mathrm{I}}\right)^{T} G_{\mathrm{I}} x^{\mathrm{II}}+\left(c^{\mathrm{I}}\right)^{T} y \\
\text { subject to } & A_{\mathrm{I}} x^{\mathrm{I}} \leq b^{\mathrm{I}}, \\
& M y+N_{\mathrm{I}} x^{\mathrm{I}}+N_{\mathrm{II}} x^{\mathrm{II}}+q \geq 0, y \geq 0, \\
& y^{T}\left(M y+N_{\mathrm{I}} x^{\mathrm{I}}+N_{\mathrm{II}} x^{\mathrm{II}}+q\right)=0 .
\end{array}
$$

Player II's problem:

$$
\begin{array}{cl}
\underset{x^{\mathrm{I}}, y}{\operatorname{minimize}} & \frac{1}{2}\left(x^{\mathrm{II}}\right)^{T} H_{\mathrm{II}} x^{\mathrm{II}}+\left(x^{\mathrm{II}}\right)^{T} G_{\mathrm{II}} x^{\mathrm{I}}+\left(c^{\mathrm{II}}\right)^{T} y \\
\text { subject to } & A_{\mathrm{II}} x^{\mathrm{II}} \leq b^{\mathrm{II}}, \\
& M y+N_{\mathrm{I}} x^{\mathrm{I}}+N_{\mathrm{II}} x^{\mathrm{II}}+q \geq 0, y \geq 0, \\
& y^{T}\left(M y+N_{\mathrm{I}} x^{\mathrm{I}}+N_{\mathrm{II}} x^{\mathrm{II}}+q\right)=0 .
\end{array}
$$


We assume that $M \in R^{m \times m}$ is a P-matrix, $H_{\nu} \in R^{m \times n_{\nu}}$ are symmetric positive definite matrices, and $G_{\nu} \in R^{n_{\nu} \times n_{-\nu}}, q \in R^{m}, c^{\nu} \in R^{m}, A_{\nu} \in R^{s_{\nu} \times n_{\nu}}, b^{\nu} \in R^{s_{\nu}}, N_{\nu} \in R^{m \times n_{\nu}}$, where $\nu=\mathrm{I}, \mathrm{II}$.

In light of the analysis about the P-matrix LCP in the previous sections, after substituting $y(x)=y\left(x^{\mathrm{I}}, x^{\mathrm{II}}\right)$ for $y$ in the objective functions of two players, we can further reformulate this EPEC as the following nonsmooth NEP:

Player I's problem:

$$
\begin{array}{ll}
\underset{x^{\mathrm{I}}}{\operatorname{minimize}} & \frac{1}{2}\left(x^{\mathrm{I}}\right)^{T} H_{\mathrm{I}} x^{\mathrm{I}}+\left(x^{\mathrm{I}}\right)^{T} G_{\mathrm{I}} x^{\mathrm{II}}+\left(c^{\mathrm{I}}\right)^{T} y(x) \\
\text { subject to } & A_{\mathrm{I}} x^{\mathrm{I}} \leq b^{\mathrm{I}} .
\end{array}
$$

Player II's problem:

$$
\begin{array}{ll}
\underset{x^{\mathrm{II}}}{\operatorname{minimize}} & \frac{1}{2}\left(x^{\mathrm{II}}\right)^{T} H_{\mathrm{II}} x^{\mathrm{II}}+\left(x^{\mathrm{II}}\right)^{T} G_{\mathrm{II}} x^{\mathrm{I}}+\left(c^{\mathrm{II}}\right)^{T} y(x) \\
\text { subject to } & A_{\mathrm{II}} x^{\mathrm{II}} \leq b^{\mathrm{II}} .
\end{array}
$$

Remark 6.1. Since we replace $y$ by $y(x)$ in the objective functions of two players, the two vector variables $\left(x^{\nu}, y\right)$ of player $\nu$ in the above EPEC reduce to one vector variable $x^{\nu}$ in the nonsmooth NEP.

Moreover, we have the following sequence of smoothed approximations to the above NEP:

Player I's problem:

$$
\begin{array}{ll}
\underset{x^{\mathrm{I}}}{\operatorname{minimize}} & \frac{1}{2}\left(x^{\mathrm{I}}\right)^{T} H_{\mathrm{I}} x^{\mathrm{I}}+\left(x^{\mathrm{I}}\right)^{T} G_{\mathrm{I}} x^{\mathrm{II}}+\left(c^{\mathrm{I}}\right)^{T} y_{\mu^{k}}(x) \\
\text { subject to } & A_{\mathrm{I}} x^{\mathrm{I}} \leq b^{\mathrm{I}} .
\end{array}
$$

Player II's problem:

$$
\begin{array}{ll}
\underset{x^{\mathrm{II}}}{\operatorname{minimize}} & \frac{1}{2}\left(x^{\mathrm{II}}\right)^{T} H_{\mathrm{II}} x^{\mathrm{II}}+\left(x^{\mathrm{II}}\right)^{T} G_{\mathrm{II}} x^{\mathrm{I}}+\left(c^{\mathrm{II}}\right)^{T} y_{\mu^{k}}(x) \\
\text { subject to } & A_{\mathrm{II}} x^{\mathrm{II}} \leq b^{\mathrm{II}} .
\end{array}
$$

By concatenating their KKT conditions, we have the following mixed CP for each $k$ :

$$
\begin{array}{r}
H_{\mathrm{I}} x^{\mathrm{I}}+G_{\mathrm{I}} x^{\mathrm{II}}+\nabla_{x^{\mathrm{I}}} y_{\mu^{k}}(x) c^{\mathrm{I}}+A_{\mathrm{I}}^{T} \lambda^{\mathrm{I}}=0, \\
H_{\mathrm{II}} x^{\mathrm{II}}+G_{\mathrm{II}} x^{\mathrm{I}}+\nabla_{x^{\mathrm{II}}} y_{\mu^{k}}(x) c^{\mathrm{II}}+A_{\mathrm{II}}^{T} \lambda^{\mathrm{II}}=0, \\
-A_{\mathrm{I}} x^{\mathrm{I}}+b^{\mathrm{I}} \geq 0, \lambda^{\mathrm{I}} \geq 0,\left(-A_{\mathrm{I}} x^{\mathrm{I}}+b^{\mathrm{I}}\right)^{T} \lambda^{\mathrm{I}}=0, \\
-A_{\mathrm{II}} x^{\mathrm{II}}+b^{\mathrm{II}} \geq 0, \lambda^{\mathrm{II}} \geq 0,\left(-A_{\mathrm{II}} x^{\mathrm{II}}+b^{\mathrm{II}}\right)^{T} \lambda^{\mathrm{II}}=0,
\end{array}
$$

where $\lambda^{\nu} \in R^{s_{\nu}}, \nu=\mathrm{I}$, II. 
It is well-known that a mixed $\mathrm{CP}$ is equivalent to a box constrained variational inequality problem (BVIP) [5]. Consequently, to deal with the above mixed CP, we consider the following BVIP: Find a vector $z=(x, \lambda) \in \mathbb{B}$ such that

$$
F_{\mu^{k}}(z)^{T}\left(z^{\prime}-z\right) \geq 0 \quad \text { for all } z^{\prime}=\left(x^{\prime}, \lambda^{\prime}\right) \in \mathbb{B}
$$

where $\mathbb{B}=\left\{z \in R^{n_{\mathrm{I}}+n_{\mathrm{II}}+s_{\mathrm{I}}+s_{\mathrm{II}}} \mid l_{i} \leq z_{i} \leq u_{i}, l_{i}=-\infty, i=1, \cdots, n_{\mathrm{I}}+n_{\mathrm{II}} ; l_{i}=0, i=\right.$ $\left.n_{\mathrm{I}}+n_{\mathrm{II}}+1, \cdots, n_{\mathrm{I}}+n_{\mathrm{II}}+s_{\mathrm{I}}+s_{\mathrm{II}} ; u_{i}=+\infty, i=1, \cdots, n_{\mathrm{I}}+n_{\mathrm{II}}+s_{\mathrm{I}}+s_{\mathrm{II}}\right\}$, and

$$
F_{\mu^{k}}(z)=\left(\begin{array}{c}
H_{\mathrm{I}} x^{\mathrm{I}}+G_{\mathrm{I}} x^{\mathrm{II}}+\nabla_{x^{\mathrm{I}}} y_{\mu^{k}}(x) c^{\mathrm{I}}+A_{\mathrm{I}}^{T} \lambda^{\mathrm{I}} \\
H_{\mathrm{II}} x^{\mathrm{II}}+G_{\mathrm{II}} x^{\mathrm{I}}+\nabla_{x^{\mathrm{II}}} y_{\mu^{k}}(x) c^{\mathrm{II}}+A_{\mathrm{II}}^{T} \lambda^{\mathrm{II}} \\
-A_{\mathrm{I}} x^{\mathrm{I}}+b^{\mathrm{I}} \\
-A_{\mathrm{II}} x^{\mathrm{II}}+b^{\mathrm{II}}
\end{array}\right)
$$

The Jacobian matrix of function $F_{\mu^{k}}(z)$ can be written as

$$
\nabla F_{\mu^{k}}(z)=\left(\begin{array}{c}
\left(\begin{array}{cc}
H_{\mathrm{I}} & G_{\mathrm{II}}^{T} \\
G_{\mathrm{I}}^{T} & H_{\mathrm{II}}
\end{array}\right)+\nabla_{x}\left(\begin{array}{c}
\nabla_{x^{\mathrm{I}}} y_{\mu^{k}}(x) c^{\mathrm{I}} \\
\nabla_{x^{\mathrm{I}}} y_{\mu^{k}}(x) c^{\mathrm{II}}
\end{array}\right), \\
\left(\begin{array}{cc}
A_{\mathrm{I}} & 0 \\
0 & A_{\mathrm{II}}
\end{array}\right), \\
0
\end{array}\right.
$$

To solve the BVIP, Kanzow and Fukushima [15] present a Newton-type method applied to the nonsmooth equation involving the natural residual of the BVIP. The algorithm uses the D-gap function to ensure global convergence of the Newton-type method. To solve the BVIP (22), we use Algorithm 3.2 in [15] with the following parameter setting:

$$
\begin{aligned}
& \alpha=0.9, \quad \beta=1.1, \quad \delta=0.6, \rho=10^{-7}, \\
& \sigma=10^{-5}, \quad p=2.1, \quad \eta=0.9, \epsilon=10^{-6} .
\end{aligned}
$$

Example 6.2. The problem data are given as follows:

$$
\begin{aligned}
& H_{\mathrm{I}}=\left(\begin{array}{cc}
3.6 & -1.2 \\
-1.5 & 2.8
\end{array}\right), H_{\mathrm{II}}=\left(\begin{array}{cc}
7.5 & -2.6 \\
-2.6 & 5.7
\end{array}\right), G_{\mathrm{I}}=\left(\begin{array}{cc}
1.1 & -1.3 \\
-2.4 & 1.6
\end{array}\right), \\
& G_{\mathrm{II}}=\left(\begin{array}{cc}
-1.2 & 2.3 \\
1.4 & -2.5
\end{array}\right), M=\left(\begin{array}{cc}
3.6 & -1.2 \\
-1.2 & 2.8
\end{array}\right), N_{\mathrm{I}}=\left(\begin{array}{cc}
2.1 & -1.3 \\
-3.4 & 2.3
\end{array}\right), \\
& N_{\mathrm{II}}=\left(\begin{array}{ll}
-5.4 & 1.6 \\
-6.2 & 2.1
\end{array}\right), q=\left(\begin{array}{c}
1.2 \\
1.6
\end{array}\right), c^{\mathrm{I}}=\left(\begin{array}{l}
-2.3 \\
-3.2
\end{array}\right), c^{\mathrm{II}}=\left(\begin{array}{c}
-2.5 \\
-2.4
\end{array}\right), \\
& A_{\mathrm{I}}=\left(\begin{array}{ll}
3.3 & -2.4
\end{array}\right), A_{\mathrm{II}}=\left(\begin{array}{ll}
-2.5 & 2.1
\end{array}\right), b^{\mathrm{I}}=-2.8, b^{\mathrm{II}}=-7.5 .
\end{aligned}
$$


Table 1. Computational Results for Example 6.1

\begin{tabular}{|c||c|c|c|c|}
\hline$\mu^{k}$ & 0.01 & 0.001 & 0.0001 & 0.00001 \\
\hline \multirow{2}{*}{$x^{k}$} & $\left(\begin{array}{c}-0.26175308 \\
0.806760257 \\
2.694216811 \\
-0.364020746\end{array}\right)$ & $\left(\begin{array}{c}-0.261753277 \\
0.806760076 \\
2.694217254 \\
-0.364019171\end{array}\right)$ & $\left(\begin{array}{c}-0.261752853 \\
0.806760137 \\
2.694217618 \\
-0.36401958\end{array}\right)$ & $\left(\begin{array}{c}-0.261753229 \\
0.806760144 \\
2.694217171 \\
-0.364018987\end{array}\right)$ \\
\hline$y^{k}$ & $\left(\begin{array}{l}7.153482977 \\
8.519043099\end{array}\right)$ & $\left(\begin{array}{c}7.153476511 \\
8.519035193\end{array}\right)$ & $\left(\begin{array}{c}7.153477701 \\
8.519037369\end{array}\right)$ & $\left(\begin{array}{c}7.153476063 \\
8.519034592\end{array}\right)$ \\
\hline D-gap & $3.43 \mathrm{e}-11$ & $4.25 \mathrm{e}-11$ & $3.34 \mathrm{e}-11$ & $4.58 \mathrm{e}-11$ \\
\hline
\end{tabular}

Table 2. Computational Results for Example 6.2

\begin{tabular}{|c||c|c|c|c|}
\hline$\mu^{k}$ & 0.01 & 0.001 & 0.0001 & 0.00001 \\
\hline \hline \multirow{2}{*}{$x^{k}$} & $\left(\begin{array}{c}-0.692667223 \\
1.016579205 \\
-0.108166204 \\
-0.464648787 \\
0.034905477 \\
0.619646979\end{array}\right)$ & $\left(\begin{array}{c}-0.692694868 \\
1.016540797 \\
-0.108194512 \\
-0.455051037 \\
0.033601554 \\
0.631711506\end{array}\right)$ & $\left(\begin{array}{c}-0.692701651 \\
1.016545116 \\
-0.108193163 \\
-0.455051025 \\
0.033580781 \\
0.631712791\end{array}\right)$ & $\left(\begin{array}{c}-0.692831978 \\
1.016420082 \\
-0.108237243 \\
-0.450723891 \\
0.032985916 \\
0.637152985\end{array}\right)$ \\
\hline$y^{k}$ & $\left(\begin{array}{l}0.361580945 \\
1.098110671 \\
0.348600029\end{array}\right)$ & $\left(\begin{array}{l}0.385396590 \\
1.252027604 \\
0.341167025\end{array}\right)$ & $\left(\begin{array}{c}0.385401922 \\
1.252031927 \\
0.341160304\end{array}\right)$ & $\left(\begin{array}{c}0.469066735 \\
1.642687333 \\
0.259482587\end{array}\right)$ \\
\hline D-gap & $\left(\begin{array}{l}9.78 \mathrm{e}-11 \\
9.52 \mathrm{e}-10\end{array}\right.$ & & $8.82 \mathrm{e}-11$ \\
\hline
\end{tabular}

Table 3. Computational Results for Example 6.3

\begin{tabular}{|c||c|c|c|c|}
\hline$\mu^{k}$ & 0.01 & 0.001 & 0.0001 & 0.00001 \\
\hline \hline \multirow{2}{*}{$x^{k}$} & $\left(\begin{array}{c}-0.716520923 \\
0.994069232 \\
-0.115604893 \\
-0.583389678 \\
0.051365820 \\
0.470423193\end{array}\right)$ & $\left(\begin{array}{c}-0.71659095 \\
0.994004031 \\
-0.115627191 \\
-0.583377728 \\
0.051365611 \\
0.470437974\end{array}\right)$ & $\left(\begin{array}{c}-0.716591707 \\
0.994003398 \\
-0.115627574 \\
-0.583377443 \\
0.051364984 \\
0.470437871\end{array}\right)$ & $\left(\begin{array}{c}-0.716591204 \\
0.994002798 \\
-0.115626892 \\
-0.583378032 \\
0.051366123 \\
0.470438807\end{array}\right)$ \\
\hline$y^{k}$ & $\left(\begin{array}{l}0.063229798 \\
0.066149112 \\
0.781141113\end{array}\right)$ & $\left(\begin{array}{c}0.063221059 \\
0.06612975 \\
0.781112525\end{array}\right)$ & $\left(\begin{array}{c}0.063221067 \\
0.066129587 \\
0.781112180\end{array}\right)$ & $\left(\begin{array}{c}0.063221092 \\
0.066129569 \\
0.781112210\end{array}\right)$ \\
\hline D-gap & $2.52 \mathrm{e}-10$ & $2.46 \mathrm{e}-10$ & $2.19 \mathrm{e}-10$ \\
\hline
\end{tabular}


The computed solutions $x^{k}=\left(x_{1}^{k, \mathrm{I}}, x_{2}^{k, \mathrm{I}}, x_{1}^{k, \mathrm{II}}, x_{2}^{k, \mathrm{II}}\right)^{T}$ and $y^{k}=\left(y_{\mu^{k}, 1}\left(x^{k}\right), y_{\mu^{k}, 2}\left(x^{k}\right)\right)^{T}$ of the sequence of smoothed NEPs with $\mu^{k}=10^{-k-1}, k=1,2,3,4$ along with the corresponding values of the D-gap functions are shown in Table 1.

We confirm that these approximate stationary Nash equilibria of smoothed NEPs converge to a B-stationary Nash equilibrium of the original NEP as $\mu^{k}$ tends to 0 . In fact, since $y(x)$ is differentiable at $x^{k}$ with $\mu^{k}=0.00001$, we can check the KKT conditions for each player's problem directly at $x=x^{k}$ as follows.

For Player I.

$$
\begin{array}{r}
H_{\mathrm{I}} x^{\mathrm{I}}+G_{\mathrm{I}} x^{\mathrm{II}}+\nabla_{x^{\mathrm{I}}} y(x) c^{\mathrm{I}}+A_{\mathrm{I}}^{T} \lambda^{\mathrm{I}}=\left(\begin{array}{c}
4.56 \mathrm{e}-006 \\
-8.14 \mathrm{e}-007
\end{array}\right), \text { with } \lambda^{\mathrm{I}}=0.597171017, \\
A_{\mathrm{I}} x^{\mathrm{I}}-b^{\mathrm{I}}=-1.00 \mathrm{e}-005 .
\end{array}
$$

For Player II.

$$
\begin{array}{r}
H_{\mathrm{II}} x^{\mathrm{II}}+G_{\mathrm{II}} x^{\mathrm{I}}+\nabla_{x^{\mathrm{II}}} y(x) c^{\mathrm{II}}+A_{\mathrm{II}}^{T} \lambda^{\mathrm{II}}=\left(\begin{array}{c}
5.07 \mathrm{e}-006 \\
-3.25 \mathrm{e}-006
\end{array}\right), \text { with } \lambda^{\mathrm{II}}=3.077495909, \\
A_{\mathrm{II}} x^{\mathrm{II}}-b^{\mathrm{II}}=1.72 \mathrm{e}-005 .
\end{array}
$$

This indicates that we can look on $x^{k}$ as an approximate B-stationary Nash equilibrium of the original NEP.

Next, we solve two EPECs where both the upper level variables and the lower level variable are three dimensional.

Example 6.3. The problem data are given as follows:

$$
\begin{aligned}
& H_{\mathrm{I}}=\left(\begin{array}{ccc}
10.0 & 3.6 & 2.7 \\
3.6 & 12.0 & -1.9 \\
2.7 & -1.9 & 15.0
\end{array}\right), H_{\mathrm{II}}=\left(\begin{array}{ccc}
12.0 & -1.2 & 3.1 \\
-1.2 & 10.0 & 2.5 \\
3.1 & 2.5 & 8.0
\end{array}\right), G_{\mathrm{I}}=\left(\begin{array}{ccc}
1.2 & 0.0 & -1.6 \\
1.3 & -2.1 & 0.0 \\
-1.2 & 1.5 & 0.3
\end{array}\right), \\
& G_{\mathrm{II}}=\left(\begin{array}{ccc}
1.2 & 0.0 & -1.5 \\
1.5 & 1.4 & 0.0 \\
-1.2 & 1.1 & -1.4
\end{array}\right), M=\left(\begin{array}{ccc}
5.6 & -1.2 & 1.5 \\
3.2 & 7.2 & -2.4 \\
-1.8 & 2.5 & 6.4
\end{array}\right), N_{\mathrm{I}}=\left(\begin{array}{ccc}
-1.1 & 0.0 & -1.2 \\
1.5 & -1.0 & -0.3 \\
-1.4 & 0.0 & 1.3
\end{array}\right) \text {, } \\
& N_{\mathrm{II}}=\left(\begin{array}{ccc}
-1.3 & 0.9 & -0.6 \\
-1.4 & 1.2 & 0.0 \\
1.5 & -0.7 & 1.4
\end{array}\right), q=\left(\begin{array}{c}
-3.2 \\
-2.5 \\
-4.8
\end{array}\right), c^{\mathrm{I}}=\left(\begin{array}{c}
-3.6 \\
-2.7 \\
-4.8
\end{array}\right), c^{\mathrm{II}}=\left(\begin{array}{c}
-3.2 \\
-2.4 \\
-4.5
\end{array}\right) \text {, } \\
& A_{\mathrm{I}}=\left(\begin{array}{ccc}
1.6 & -1.3 & -1.2 \\
1.2 & -1.7 & 1.3
\end{array}\right), A_{\mathrm{II}}=\left(\begin{array}{ccc}
1.3 & -1.5 & -1.2 \\
1.8 & 1.2 & -1.3
\end{array}\right), b^{\mathrm{I}}=\left(\begin{array}{c}
-2.3 \\
-2.7
\end{array}\right), b^{\mathrm{II}}=\left(\begin{array}{l}
-1.4 \\
-1.6
\end{array}\right) \text {. }
\end{aligned}
$$


Example 6.4. The problem data are the same as those in Example 6.3 except that $c^{\mathrm{I}}, c^{\mathrm{II}}$ and $q$ are given as follows:

$$
c^{\mathrm{I}}=\left(\begin{array}{c}
-3.6 \\
2.7 \\
-4.8
\end{array}\right), c^{\mathrm{II}}=\left(\begin{array}{c}
3.2 \\
-2.4 \\
4.5
\end{array}\right), q=\left(\begin{array}{c}
-3.2 \\
2.5 \\
-4.8
\end{array}\right) .
$$

The computed solutions $x^{k}=\left(x_{1}^{k, \mathrm{I}}, x_{2}^{k, \mathrm{I}}, x_{3}^{k, \mathrm{I}}, x_{1}^{k, \mathrm{II}}, x_{2}^{k, \mathrm{II}}, x_{3}^{k, \mathrm{II}}\right)^{T}$ and $y^{k}=\left(y_{\mu^{k}, 1}\left(x^{k}\right), y_{\mu^{k}, 2}\left(x^{k}\right)\right.$, $\left.y_{\mu^{k}, 3}\left(x^{k}\right)\right)^{T}$ of the sequence of smoothed NEPs with $\mu^{k}=10^{-k-1}, k=1,2,3,4$ along with the values of D-gap functions are shown in Table 2 and Table 3.

For these two examples, we may observe the similar properties to those of Example 6.2. As $\mu^{k}$ tends to 0 , we confirm that these approximate stationary Nash equilibria of smoothed NEPs also converge to a B-stationary Nash equilibrium of the original NEP. In fact, in Example 6.3, since $y(x)$ is differentiable at $x^{k}$ with $\mu^{k}=0.00001$, we can check the KKT conditions for each player's problem directly at $x=x^{k}$ as follows.

For Player I.

$$
\begin{array}{r}
H_{\mathrm{I}} x^{\mathrm{I}}+G_{\mathrm{I}} x^{\mathrm{II}}+\nabla_{x^{\mathrm{I}}} y(x) c^{\mathrm{I}}+A_{\mathrm{I}}^{T} \lambda^{\mathrm{I}}=\left(\begin{array}{c}
1.76 \mathrm{e}-006 \\
-1.49 \mathrm{e}-006 \\
-1.49 \mathrm{e}-007
\end{array}\right), \text { with } \lambda^{\mathrm{I}}=\left(\begin{array}{c}
0.316295946 \\
5.198888467
\end{array}\right), \\
A_{\mathrm{I}} x^{\mathrm{I}}-b^{\mathrm{I}}=\left(\begin{array}{c}
7.42 \mathrm{e}-006 \\
2.09 \mathrm{e}-005
\end{array}\right) .
\end{array}
$$

For Player II.

$$
\begin{array}{r}
H_{\mathrm{II}} x^{\mathrm{II}}+G_{\mathrm{II}} x^{\mathrm{I}}+\nabla_{x^{\mathrm{II}}} y(x) c^{\mathrm{II}}+A_{\mathrm{II}}^{T} \lambda^{\mathrm{II}}=\left(\begin{array}{c}
2.65 \mathrm{e}-006 \\
-1.68 \mathrm{e}-008 \\
-1.51 \mathrm{e}-006
\end{array}\right), \text { with } \lambda^{\mathrm{II}}=\left(\begin{array}{c}
3.355974139 \\
0.829159978
\end{array}\right), \\
A_{\mathrm{II}} x^{\mathrm{II}}-b^{\mathrm{II}}=\left(\begin{array}{c}
-3.51 \mathrm{e}-006 \\
-1.88 \mathrm{e}-005
\end{array}\right) .
\end{array}
$$

This indicates that we can look on $x^{k}$ as an approximate B-stationary Nash equilibrium of the original NEP.

\section{Conclusions}

In this paper, we have proposed an approach to deal with a special class of EPECs, where the players share a parametric P-matrix linear complementarity constraint. Exploiting the good 
properties of a P-matrix, we have reformulated the EPEC as an equivalent NEP with nonsmooth objective functions. We have further employed a smoothing method to construct a sequence of smoothed NEPs to approximate the original NEP. We have established some convergence results about the approximate global Nash equilibria and approximate stationary Nash equilibria. We have confirmed the validity of the proposed approach through numerical experiments.

\section{References}

[1] Chen, X., Fukushima, M.: A smoothing method for a mathematical program with Pmatrix linear complementarity constraints. Comput. Optim. Appl. 27, 223-246 (2004)

[2] Clarke, F.H.: Optimization and Nonsmooth Analysis. SIAM: Philadelphia (1990)

[3] Cottle, R.W., Pang, J.S., Stone, R.E.: The Linear Complementarity Problem. Academic Press: New York (1992)

[4] Facchinei, F., Jiang, H., Qi, L.: A smoothing method for mathematical programs with equilibrium constraints. Math. Program. 85, 107-134 (1999)

[5] Facchinei, F., Pang, J.S.: Finite-Dimensional Variational Inequalities and Complementarity Problems. Volumes I and II, Springer, New York (2003)

[6] Fischer, A.: A special Newton-type optimization method. Optimization. 24, 269-284 (1992)

[7] Fukushima, M., Luo, Z.Q., Pang, J.S.: A globally convergent sequential programming algorithm for mathematical programs with linear complementarity constraints. Comput. Optim. Appl. 10, 5-30 (1998)

[8] Fukushima, M., Pang, J.S.: Convergence of a smoothing continuation method for mathematical programs with complementarity constraints. In: Théra, M., Tichatschke, R. (ed.) Ill-posed Variational Problems and Regularization Techniques. Lect. Notes Econ. Math. 477, Springer-Verlag, Berlin/Heidelberg, 99-110 (1999)

[9] Gürkan, G., Pang, J.S.: Approximations of Nash equilibria. Math. Program. Ser. B. 117, 223-253 (2009)

[10] Hobbs, B.F., Metzler, C., Pang, J.S.: Strategic gaming analysis for electric power networks: An MPEC approach. IEEE Trans. Power Syst. 15, 638-645 (2000) 
[11] Hu, X., Ralph, D.: Using EPECs to model bilevel games in restructured electricity markets with locational prices. Oper. Res. 55, 809-827 (2007)

[12] Jofré, A., Wets, R.J.-B.: Variational convergence of bivariate functions: lopsided convergence. Math. Program. Ser. B. 116, 275-295 (2009)

[13] Kall, P.: Approximation to optimization problems: An elementary review. Math. Oper. Res. 11, 9-18 (1986)

[14] Kanzow, C.: Some noninterior contination methods for linear complementarity problem. SIAM J. Matrix Anal. Appl. 17, 851-868 (1996)

[15] Kanzow, C., Fukushima, M.: Solving box constrained variational inequalities by using the natural residual with D-gap function globalization. Oper. Res. Lett. 23, 45-51 (1998)

[16] Luo, Z.Q., Pang, J.S., Ralph, D.: Mathematical Programs with Equilibrium Constraints. Cambridge University Press, Cambridge, UK (1996)

[17] Nash, J.F.: Equilibrium points in $n$-person games. Proc. Nat. Acad. Sci. U.S.A. 36, 48-49 (1950)

[18] Nash, J.F.: Non-cooperative games. Ann. Math. 54, 286-295 (1951)

[19] Outrata, J., Kočvara, M., Zowe, J.: Nonsmooth Approach to Optimization Problems with Equilibrium Constraints: Theory, Applications and Numerical Results. Kluwer Academic Publishers, Dordrecht, The Netherlands (1998)

[20] Pang, J.S., Fukushima, M.: Quasi-variational inequalities, generalized Nash equilibria, and multi-leader-follower games, Comput. Manage. Sci. 2, 21-56 (2005); Erratum, ibid. 6, 373-375 (2009)

[21] Rockafellar, R.T., Wets, R.J.-B.: Variational Analysis. Springer, Berlin (1998)

[22] Stackelberg, H.V.: The Theory of Market Economy. Oxford University Press (1952) 